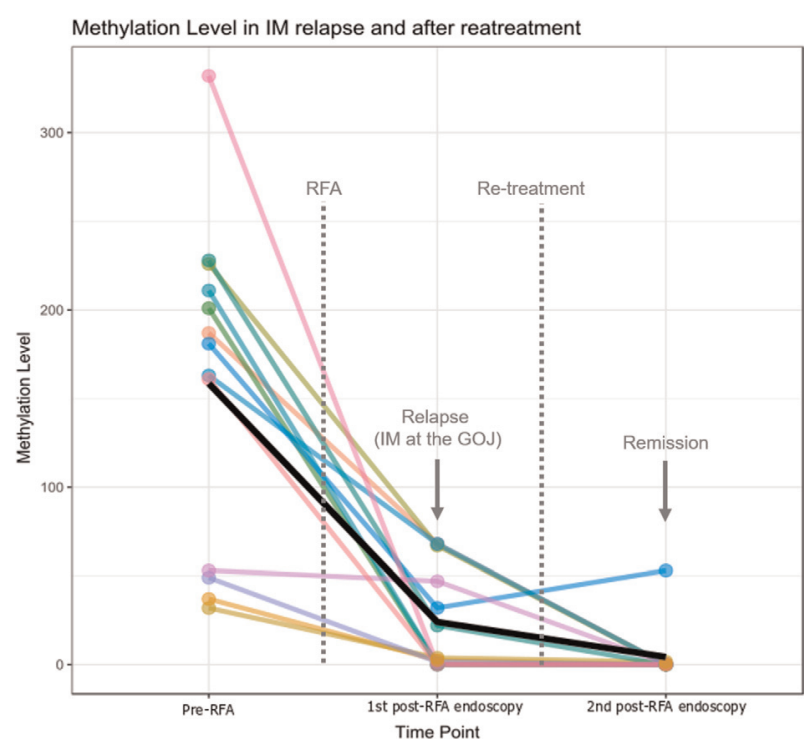

Abstract 0TU-17 Figure 1 Methylation levels in oesophageal biopsies before radio-frequency ablation, at relapse within the gastrooesophageal junction and after re-treatment

(17.1\%) had IM score 2-3, with corresponding mean methylation levels of $2.4 \%$ and $35.5 \%$, respectively $(P=0.018)$. In patients with GOJ-IM, who received RFA re-treatment, the Meth-Score decreased from $24 \%$ to $4.2 \%(P=0.027)$ as presented in figure 1.

Conclusions The methylation panel can differentiate grades of dysplasia in Barrett's and significantly correlates with the extent of IM at the GOJ. We propose the Meth-Score as an objective measure of residual and recurrent disease following RFA.

GOJ, gastro-oesophageal junction; IM, intestinal metaplasia; RFA, radio-frequency ablation.

\section{OTU-18 USING X-RAY PHASE CONTRAST IMAGING TO IDENTIFY OESOPHAGEAL PATHOLOGY}

${ }^{1}$ Paul Wolfson*, ${ }^{2}$ Jinxing Jiang, ${ }^{1}$ Ash Wilson, ${ }^{2}$ Lorenzo Massimi, ${ }^{3}$ Marco Novelli, ${ }^{1}$ Laurence Lovat, ${ }^{2}$ Sandro Olivo. 'University College London, Division of Surgery and Interventional Science, London, UK; ${ }^{2}$ University College London, Department of Medical Physics and Biomedical Engineering, London, UK; ${ }^{3}$ University College London, Department of Pathology, London, UK

\subsection{6/gutjnl-2019-BSGAbstracts.253}

Introduction Oesophageal cancer is the 5th commonest cause of cancer death in the UK. It accounts for 9,000 new cancer cases every year. Most oesophageal cancers are diagnosed at a late stage. Survival data shows that patients with early disease have a $37.5 \% 5$ year survival compared to $8.4 \%$ and $4.9 \%$ for those with regional or distant disease. ${ }^{1}$

Histological diagnosis of oesophageal pathology is an expensive and time consuming process with high inter observer variability. X-ray imaging is inexpensive and has not previously been used for imaging of soft tissue due to poor contrast resolution. X-ray phase contrast imaging (XPCI) uses refraction of $\mathrm{x}$-rays as they pass through a tissue rather than attenuation, which provides much higher soft tissue contrast ${ }^{2}$. It can also be tuned to have a depth resolution of between 5 $\mu \mathrm{m}$ and $10 \mathrm{~mm}$. This may allow for easy assessment of extent of disease infiltration.
Aims We aimed to use XPCI to image four types of oesophageal mucosa, normal squamous, non-dysplastic Barrett's oesophagus, Barrett's oesophagus with high-grade dysplasia and oesophageal adenocarcinoma.

Methods Following ethical approval, twelve biopsies were obtained both from patients attending for endoscopy at UCLH as well as from the CRUK Cancer Centre Biobank. Biopsies were imaged at the Diamond Synchrotron in beamline i13 using an XPCI technique called free space propagation. We reconstructed the images using MATLAB ${ }^{\circledast}$ software. These CT images were compared to histology from matched biopsies taken at the time of endoscopy or the original histological diagnosis if biobank tissue was used.

Results We successfully reconstructed CT images for all four tissue types achieving a resolution of $\sim 5$ microns. We were able to identify normal layered squamous mucosa as well as changes within the structure of the biopsies including glands and goblet cells. The four tissue types appear to have quite different morphology. In addition, the three dimensional tissue structure could be clearly identified, with cancer being the most disorganised.

This is the first time that XPCI has been used to image oesophageal biopsies. We have demonstrated the feasibility of the technique and the possibility of obtaining high resolution images which mimic histology with the extra benefit of demonstrating three dimensional structure.

\section{REFERENCES}

1. "Oesophageal cancer incidence statistics | Cancer Research UK." [Online]. Available: https://www.cancerresearchuk.org/health-professional/cancer-statistics/statistics-by-cancer-type/oesophageal-cancer/incidence\#tref-5. [Accessed: 29-Jan-2019].

2. Olivo A, Gkoumas $S$, Endrizzi $M$, et al. Low-dose phase contrast mammography with conventional x-ray sources. Medical Physics 2013;40(9);90701. doi:10.1118/ 1.4817480

\section{OTU-20 REDUCED STRICTURE RATES WITH A NOVEL HALO 360 RADIOFREQUENCY REGIME FOR BARRETT'S DYSPLASIA}

${ }^{1}$ E Saffouri*, ${ }^{1} R$ Haddock, ${ }^{1} \mathrm{TJ}$ Berriman, ${ }^{2} \mathrm{~J}$ Pugmire, ${ }^{1} \mathrm{G}$ Fullarton, ${ }^{1} \mathrm{AJ}$ Morris. ${ }^{1}$ Department of Gastroenterology and Upper GI Surgery, Glasgow Royal Infirmary, Glasgow, UK; ${ }^{2}$ University of Glasgow, Glasgow, UK

\subsection{6/gutjnl-2019-BSGAbstracts.254}

Introduction In the UK, radiofrequency ablation (RFA) is established as treatment of choice for flat oesophageal neoplasia or after removal of focal lesions by endoscopic mucosal resection (EMR) to eradicate Barrett's mucosa. Standard practice is treatment with the HALO 360 Express RFA catheter. A specific complication of RFA is oesophageal stricture development. The UK national RFA registry has quoted a $11-17 \%$ rate of strictures requiring dilatation, with higher rates in patients treated with $12 \mathrm{~J}$ rather than $10 \mathrm{~J}$ paired ablations $(\mathrm{p}<0.01)$. Two 10J ablations, separated by a cleaning step requiring removal of the catheter device, is now standard of care. The cleaning step is a time-consuming part of the procedure and can be poorly tolerated.

Methods In December 2017, we adapted our practice to include irrigation with $30 \mathrm{cc}$ normal saline between $10 \mathrm{~J}$ ablations as a cooling phase during the HALO 360 express procedure and removed the cleaning phase between ablations. We have audited patient and disease demographics and outcomes data, especially oesophageal stricture rate, for all patients who had first HALO 360 express between 1/12/16-1/12/18 in our hospital. This represents 12 months before and after technique 